\title{
Schon Gewichthalten hilft gegen Diabetes
}

\author{
Nichts geht über Abspecken - aber schon wenn alle Erwachsenen ihr Körpergewicht über zehn Jahre \\ konstant hielten, würde auf Populationsebene mindestens ein Fünftel aller Diabetesfälle verhindert.
}

Für die schwedische VästerbottenStudie wurden $>$ 33.000 Personen im Alter von 30-60 Jahren zwischen 1990 und 2013 fortlaufend untersucht. In einer multivariaten logistischen Regressionsanalyse errechnete man, wie sich eine Veränderung des BMI über zehn Jahre auf die Inzidenz des Diabetes mellitus auswirkt.

Der mittlere BMI stieg in dieser Zeit von $25 \pm 3,6$ auf $26,3 \pm 4,1 \mathrm{~kg} / \mathrm{m}^{2}$. 29,1\% der Teilnehmer hatten ihr Gewicht in einem Bereich von $\pm 3 \%$ gehalten, $56,6 \%$ hatten zugenommen, $14,2 \%$ abgenommen. Bei 1.087 Teilnehmern (3,3\%) manifestierte sich ein Diabetes mellitus. Mehr als ein Drittel dieser Personen hatte bereits bei Studieneintritt eine gestörte Glukosetoleranz oder einen Nüchternglukosewert im Grenzbereich.
Eine BMI-Zunahme von $>1 \mathrm{~kg} / \mathrm{m}^{2}$ bedeutete ein höheres Risiko für einen neu aufgetretenen Diabetes als ein konstant gehaltenes Gewicht (Odds Ratio 1,52, 95\%-Konfidenzintervall 1,32-1,74), wobei sich keine Anhaltspunkte für einen nicht-linearen Zusammenhang fanden. Rechnerisch ergab sich, dass auf Populationsebene 21,9\% der neuen Diabetesfälle auf diese Gewichtszunahme zurückgingen. Ein mäßiger BMI-Rückgang von $1-2 \mathrm{~kg} / \mathrm{m}^{2}$ verringerte dagegen das Risiko für einen neu aufgetretenen Diabetes mellitus um 28\%.

Würde es also gelingen, den durchschnittlichen BMI von Personen im mittleren Lebensalter über zehn Jahre zwischen 1 und $2 \mathrm{~kg} / \mathrm{m}^{2}$ zu verringern, so könnten zwei von fünf Diabetesfälle verhindert werden. Das wären im
Schnitt nur 3-6 kg pro Person. Bei lebenslanger Betrachtung wäre es immerhin einer von fünf Fällen.

- Feldman AL et al. Impact of weight maintenance and loss on diabetes risk and burden: a population-based study in 33,184 participants. BMC Public Health. 2017;17:170-80

\section{KOMMENTAR}

Die Untersuchung zeigt, welch großes Potenzial für die Diabetesprävention in der Reduzierung des Körpergewichts liegt. Statt Milliarden für die Therapie des manifesten Diabetes und seiner Komplikationen auszugeben, müssten sich alle Anstrengungen auf das Ernährungsverhalten und die körperliche Aktivität konzentrieren. Leider hat das mit Medizin sehr wenig zu tun - und Geld ist damit kaum zu verdienen.

Prof. Dr. med. H. S. FüeßI

\section{Sweet-Syndrom bei rheumatoider Arthritis}

Eine 52-jährige Frau mit rheumatoider Arthritis hatte vor einem Monat erstmals mehrere schmerzhafte, rote Flecken an beiden Handflächen und mehreren Fingern bemerkt. Seit drei Jahren bestand eine Dauertherapie mit Sulfasalazin und Azathioprin, vor ein-

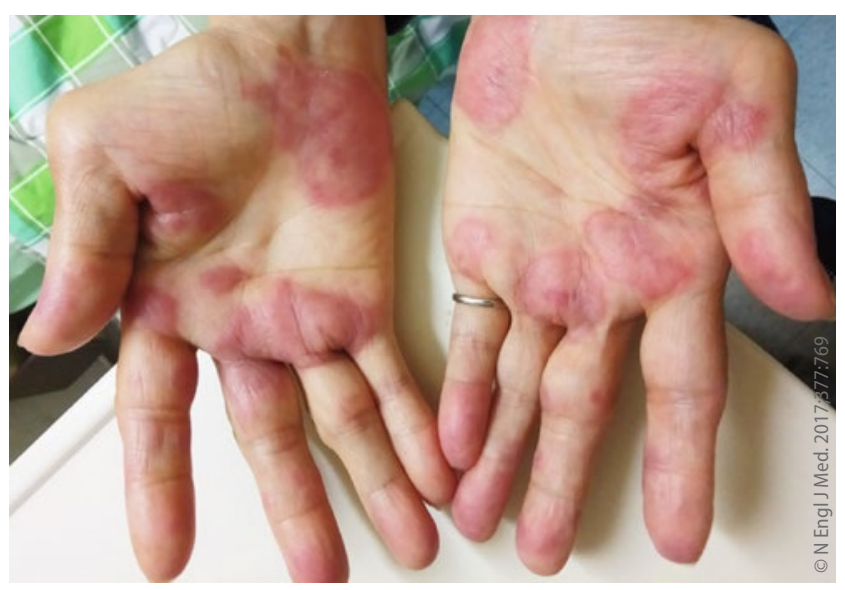

Rote Flecken an Handflächen und Fingern einer Rheumatikerin. einhalb Jahren war sie über sechs Monate hinweg mit Etanercept behandelt worden. Zusätzlich zu den Hautveränderungen ergab die Untersuchung typische Veränderungen einer fortgeschrittenen rheumatoiden Arthritis wie eine Ulnardeviation der Langfinger und eine Z-Deformität beider Daumen. Es bestand kein Fieber. Die BKS war mit $50 \mathrm{~mm} / \mathrm{h}$ deutlich erhöht, der CRP-Wert lag bei $20,2 \mathrm{mg} / \mathrm{l}$ (Normalwert <3,5), die Leukozytenzahl betrug 3.670/ $\mu$ l mit $87 \%$ Neutrophilen.

In der Hautbiopsie zeigte sich ein papilläres Hautödem mit perivaskulären und interstitiellen lympohistiozytären und neutrophiIen Infiltraten, die mit einer akuten febrilen neutrophilen Dermatose, einem Sweet-Syndrom, vereinbar waren. Differenzialdiagnostisch wären in diesem Fall eine Vaskulitis, ein Arzneimittelexanthem oder Hautinfektionen zu erwägen. Nach einer kurzen Stoßtherapie mit Prednisolon bildeten sich die Hautveränderungen rasch zurück. Auch im Verlauf des nachfolgenden Jahres traten sie nicht mehr auf. Das Behandlungsregime der rheumatoiden Arthritis blieb unverändert.

Prof. Dr. med. H. S. FüeßI

- Huang ZH, Chen HC. Sweet's syndrome in a patient with rheumatoid arthritis. NEnglJMed. 2017;377:769 